\title{
Article \\ Self-Consistent Energy Normalization for Quasistatic Reactor Calculations
}

\author{
David P. Griesheimer *, Steven J. Douglass and Mark H. Stedry
}

Naval Nuclear Laboratory, West Mifflin, PA 15122, USA; steven.douglass@unnpp.gov (S.J.D.); mark.stedry@unnpp.gov (M.H.S.)

* Correspondence: david.griesheimer@unnpp.gov

check for updates

Citation: Griesheimer, D.P.;

Douglass, S.J.; Stedry, M.H.

Self-Consistent Energy Normalization for Quasistatic Reactor Calculations. J. Nucl. Eng. 2021, 2, 215-224. https:// doi.org/10.3390/jne2020020

Academic Editors: Eugene Shwageraus and Patrick Blaise

Received: 29 September 2020

Accepted: 28 May 2021

Published: 2 June 2021

Publisher's Note: MDPI stays neutral with regard to jurisdictional claims in published maps and institutional affiliations.

Copyright: (c) 2021 by the authors. Licensee MDPI, Basel, Switzerland. This article is an open access article distributed under the terms and conditions of the Creative Commons Attribution (CC BY) license (https:// creativecommons.org/licenses/by/ $4.0 /)$.

\begin{abstract}
Use of the quasistatic ( $\left.k_{\text {eff }}\right)$ approximation for producing steady-state solutions for noncritical fission systems is known to result in an imbalance between energy release and deposition within the system. In this paper, we formally quantify this imbalance and present a self-consistent energy normalization technique that preserves nuclear energy release per reaction, as well as enforces energy balance between release and deposition mechanisms, regardless of the criticality state of the system. The proposed technique is straightforward to implement in any type of transport solver through the use of a simple energy rebalance factor. Theoretical and numerical results are presented that demonstrate the energy deposition bias for non-critical systems and the effectiveness of the proposed energy normalization technique.
\end{abstract}

Keywords: energy deposition; nuclear reactor; power normalization

\section{Introduction}

Many types of reactor calculations, such as depletion and thermal feedback, require neutron flux distributions that are properly normalized to the specific power level of the reactor. This power normalization is commonly performed by assuming a fixed energy release per fission and scaling the flux uniformly throughout the reactor in order to achieve the specified power level. More recently, detailed reaction-specific energy release data have been used to improve the accuracy of the power normalization process by accounting for changes in fission energy release throughout reactor lifetime [1,2]. These changes are due to variations in the neutron spectrum and reactor material compositions that occur over time. Accounting for changes in fission energy release during operation helps to limit the accumulation of errors in fluence, total reactor power output, and depleted/transmuted nuclide inventories, especially at high burnup (end-of-cycle) conditions.

Detailed power normalization methods typically rely on nuclide-specific energy release data provided as raw nuclear data. For example, the Evaluated Nuclear Data File (ENDF) format provides energy release per fission (MT 458) and kinetic energy released in material (KERMA, MT 300) data by nuclide, which can be used to estimate the energy released per neutron reaction [3]. These data can be used to estimate the total rate of energy release in the reactor, which, in turn, can be used to infer the energy deposition rate based on conservation of energy within the system. Unfortunately, techniques based on conservation of energy are only guaranteed to work for critical reactors where neutron production and loss are balanced. Flux solutions for non-critical reactors are obtained via the quasistatic approximation, where the neutron production rate is artificially modified by a factor $1 / k_{\text {eff }}$ in order to force a solution to the steady-state neutron balance equation. This common, but non-physical, adjustment results in a significant, and often overlooked, effect on energy deposition rates used for high-fidelity power normalization.

In this paper, we provide a rigorous interpretation of energy balance for a non-critical reactor and present an energy renormalization scheme that preserves energy release per reaction and enforces balance between energy release and deposition, regardless of the 
multiplication factor for the system. The proposed renormalization technique is straightforward to implement in any radiation transport solver and can be used for both local and global energy deposition edits.

\section{Quasistatic Energy Balance}

In order to rigorously define detailed energy balance equations for a reactor, it is first necessary to establish a consistent nomenclature. In this work, the variable $Q$ is used to denote energy release from a nuclear reaction and $E$ refers to the energy of an individual neutron or photon. For nuclear reactions, the variable $E^{\prime}$ refers to the pre-reaction energy of the incident particle, while $E$ indicates the post-reaction energy. The non-italicized variables $Q$ and E signify total energy release and transfer, respectively, which are integrated over space as well as the energy for specified reaction types. Subscripts on variables are used to indicate the incident particle type (neutron (n) or photon $(\gamma)$ ), while superscripts denote the form of energy emitted by the reaction. The 'total' superscript is used to refer to the total energy emitted from a reaction, regardless of form, while the superscript 'local' refers to emitted energy that is deposited at the location of the reaction, typically through charged particle emission (recoil nuclei, $\alpha, \beta$-, etc.) or non-ionizing direct transfer of kinetic energy. Note that charged particle transport effects are not taken into account in this analysis, and all charged particles are assumed to deposit their kinetic energy locally, except when secondary radiation emission (bremsstrahlung, annihilation, electron relaxation) is specifically included.

We begin with the basic premise that, under steady-state (or quasistatic) conditions, the total energy released in the reactor must be equal to the sum of the energy deposited in the reactor and the energy leaking out of the reactor $\left(E_{\text {released }}=E_{\text {deposited }}+E_{\text {leak }}\right)$. This fundamental balance equation can be further expanded by considering the various energy release and deposition processes that occur as neutrons slow-down and produce fissions in a reactor:

$$
\mathrm{E}_{\mathrm{n}, \text { birth }}+\mathrm{Q}_{\text {indirect }}+\mathrm{Q}_{\text {fission }}=\left(\mathrm{E}_{\mathrm{n}, \mathrm{fission}}^{\text {total }}+\mathrm{E}_{\mathrm{n}, \text { indirect }}^{\text {total }}\right)+\mathrm{E}_{\mathrm{n}, \text { leakage }}
$$

where $E_{n, b i r t h}$ is the total birth energy of all neutrons produced in fission, $E_{n, l e a k a g e}$ is the total energy of neutrons that leak out of the reactor, $\mathrm{Q}_{\text {fission }}$ is the total energy released by fission reactions, $Q_{\text {indirect }}$ is the total energy released by non-fission nuclear reactions (capture and inelastic scattering), and $E_{n, f i s s i o n}^{\text {total }}$ and $E_{n, \text { indirect }}^{\text {total }}$ are the total energy deposited within the reactor during fission and non-fission reactions, respectively.

Equation (1) is based on mass-energy balance for neutrons in the reactor, but it does not consider the actual mechanics of energy deposition reactions, nor the effects (or subsequent transport) of secondary radiations produced. In fact, neutron energy deposition results in the transfer of neutron kinetic energy into other forms of radiation, such as photons, neutrinos, and/or charged particles.

In order to consider the effects of secondary radiation transport, we break the fission and non-fission (indirect) energy deposition terms into specific 'local' and 'non-local' components, as:

$$
\begin{aligned}
E_{n, \text { birth }}+Q_{\text {indirect }} & +Q_{\text {fission }}=\left(E_{n, \text { fission }}^{\text {local }}+E_{n, \text { fission }}^{\text {neutron }}+E_{n, \text { fission }}^{\text {neutrino }}+E_{n, \text { fission }}^{\gamma}\right) \\
& +\left(E_{n, \text { indirect }}^{\text {local }}+E_{n, \text { indirect }}^{\gamma}\right)+E_{n, \text { leakage }}
\end{aligned}
$$

Note that the non-local component for fission includes emission of neutrons, neutrinos, and photons, while the non-local component for non-fission (indirect) reactions only includes photons. This is because emission of neutrons in non-fission reactions is traditionally treated as scattering and neutrino emission is accounted for by decreasing the sensible $Q$-value for the reaction. Local energy deposition includes fission fragment and $\beta$-release for fission reactions, and recoil nuclei and charged particle emission for non-fission reactions. Energy released as neutrino and photon radiation will be transported 
away from the original neutron interaction location and deposit energy elsewhere in the problem. As a result, separate energy balance equations that relate energy release and deposition are required for both of these secondary radiation types. Neutrinos have an extremely small probability of interacting within a reactor, which leads to the trivial neutrino balance relationship

$$
\mathrm{E}_{\text {neutrino,birth }}=\mathrm{E}_{\text {neutrino,leak} \text {. }}
$$

However, photons interact and deposit energy within the reactor, as described by the photon balance equation:

$$
\mathrm{E}_{\gamma, \text { birth }}=\mathrm{E}_{\gamma, \text { indirect }}^{\text {local }}+\mathrm{E}_{\gamma, \text { leak }}
$$

where $E_{\gamma \text {,indirect }}^{\text {local }}$ is the local energy deposition due to all photon interactions. Note that this balance equation implicitly assumes that the $Q$-value for photon interactions is zero, which implies that there is no nuclear energy released through these interactions. The relationship between the various energy release, deposition, and conversion mechanisms for all coupled radiation types is illustrated in Figure 1.

\section{Fission Energy Cycle}

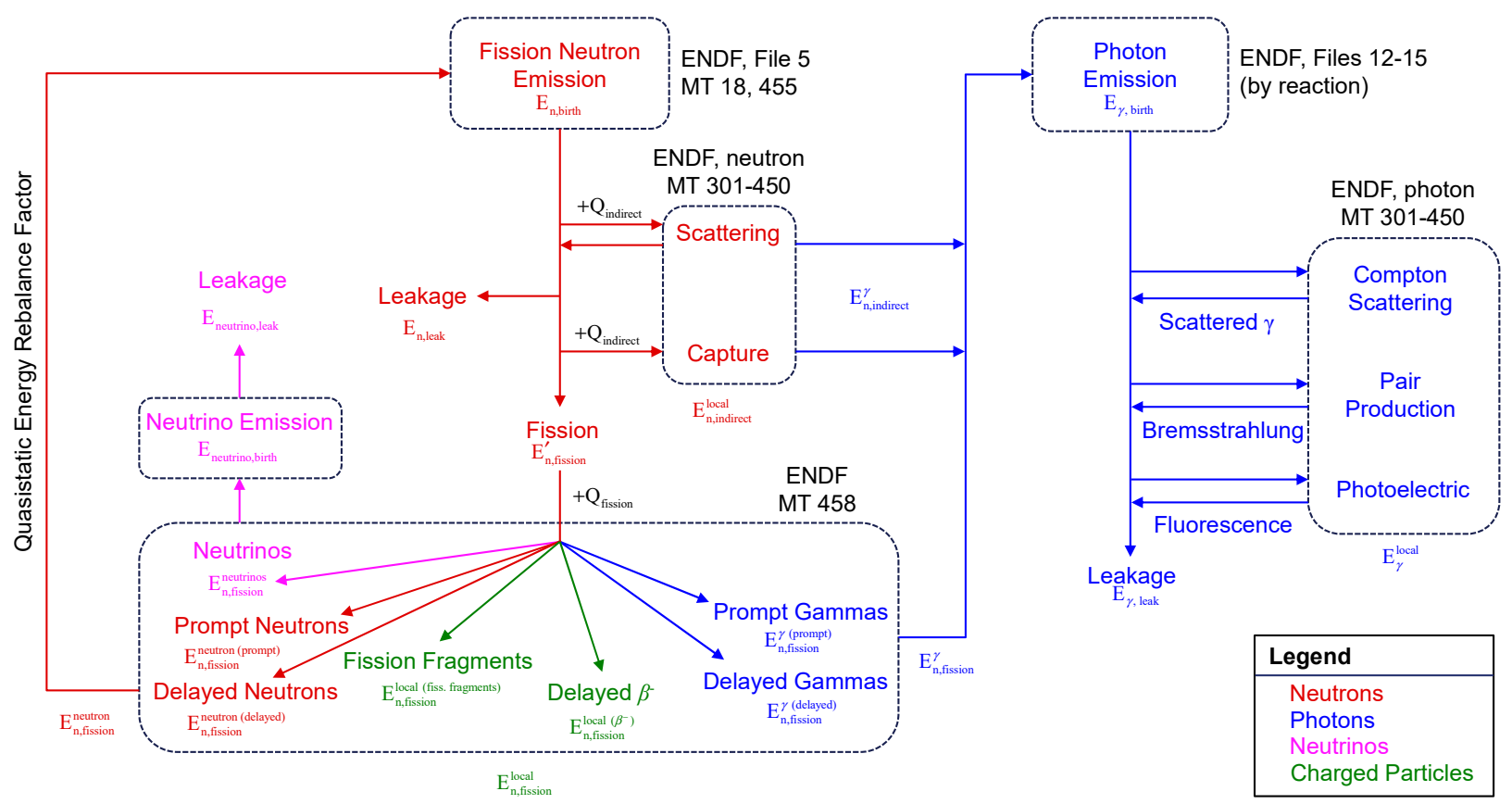

Figure 1. Illustration of energy release, deposition, and conversion mechanisms for neutrons, photons, charged particles, and neutrinos in a nuclear reactor. Evaluated Nuclear Data File (ENDF) reaction numbers (i.e., MT numbers) associated with energy release/deposition are listed for each reaction category.

Equation (2), along with the auxiliary energy balance equations for neutrinos (Equation (3)) and photons (Equation (4)), describes the total energy balance for all forms of radiation within a reactor. Under steady-state conditions (or the quasistatic assumption) these balance equations are coupled through the following relationships,

$$
\begin{gathered}
\mathrm{E}_{\mathrm{n}, \text { birth }}=\mathrm{E}_{\mathrm{n}, \mathrm{fission}{ }^{\text {neutron }}} \\
\mathrm{E}_{\text {neutrino,birth }}=\mathrm{E}_{\mathrm{n}, \text { fission }}^{\text {neutrino }} \\
\mathrm{E}_{\gamma, \text { birth }}=\mathrm{E}_{\mathrm{n}, \text { fission }}^{\gamma}+\mathrm{E}_{\mathrm{n}, \text { indirect' }}^{\gamma}
\end{gathered}
$$


which require that the energy of secondary radiation produced is equal to the energy of the corresponding radiation type released during neutron interactions (fission, capture, and scattering).

For non-critical reactors, the neutron balance condition in Equation (5) is not satisfied naturally. This can be shown by writing the total energy release terms as reaction rate integrals,

$$
\begin{aligned}
\mathrm{E}_{\mathrm{n}, \text { birth }} & =\left\langle\left\{\frac{1}{k_{\mathrm{eff}}} \bar{E}_{\mathrm{n}}\left(E_{\mathrm{n}}^{\prime}\right)\right\} v \Sigma_{\mathrm{f}}\left(\vec{r}, E_{\mathrm{n}}^{\prime}\right) \phi_{\mathrm{n}}\left(\vec{r}, E_{\mathrm{n}}^{\prime}\right)\right\rangle_{E_{\mathrm{n},}^{\prime} \vec{r}^{\prime}} \\
\mathrm{E}_{\mathrm{n}, \text { fission }}^{\text {neutron }} & =\left\langle\left\{\frac{h_{\mathrm{n}, \text { fission }}^{\text {neutron }}\left(E_{\mathrm{n}}^{\prime}\right)}{v\left(\vec{r}, E_{\mathrm{n}}^{\prime}\right)}\right\} v \Sigma_{\mathrm{f}}\left(\vec{r}, E_{\mathrm{n}}^{\prime}\right) \phi_{\mathrm{n}}\left(\vec{r}, E_{\mathrm{n}}^{\prime}\right)\right\rangle_{E_{\mathrm{n},}^{\prime}, \vec{r}},
\end{aligned}
$$

where $h_{\mathrm{n} \text {,fission }}^{\text {neutron }}$ is the energy released in fission as neutrons, $k_{\text {eff }}$ is the $k$-eigenvalue quasistatic balance factor (applied to the number of neutrons released per fission to enforce neutron balance in non-critical systems), $\bar{E}_{\mathrm{n}}\left(E_{\mathrm{n}}^{\prime}\right)$ is the average fission neutron birth energy, and angle brackets denote integration over the specified variables. In order for the fission neutron birth energy to match the neutron energy released in fission (Equation (5)) it follows that the factors in braces in Equations (8) and (9), each representing the average birth energy of a fission neutron, must be equal. However, Equation (8) shows that, for a non-critical reactor $\left(k_{\text {eff }} \neq 1\right)$, the true average fission neutron birth energy (obtained from the first moment of the fission spectrum) must be scaled by a factor of $1 / k_{\text {eff }}$, in order to preserve the energy balance. The need for this correction factor stems from the quasistatic approximation itself, where the number of neutrons released per fission is adjusted by $1 / k_{\text {eff }}$ to enforce neutron (mass) balance in the system. When the number of neutrons released per fission is adjusted, it follows that the total birth energy of neutrons will also change unless the average birth energy of fission neutrons is also adjusted by the same factor. However, changing the birth energy of fission neutrons will affect neutron transport, leading to undesired changes in the neutron flux and reaction rate distributions throughout the core.

In order to preserve energy balance without affecting the neutron transport process, it is necessary to distinguish between the energy of the neutron used to evaluate nuclear cross sections (the transport energy) and the kinetic energy carried by, and ultimately deposited by, the neutron (the deposition energy). The neutron transport energy is governed by the fission spectrum and scattering kernels for the materials present in the reactor and is not influenced by the multiplication factor $\left(k_{\text {eff }}\right)$ for the reactor. The neutron deposition energy represents the fraction of the transport energy available for deposition or leakage. The deposition energy for a neutron is defined as the transport energy of the neutron multiplied by the ratio of fission neutron energy release to the cumulative fission neutron birth energy:

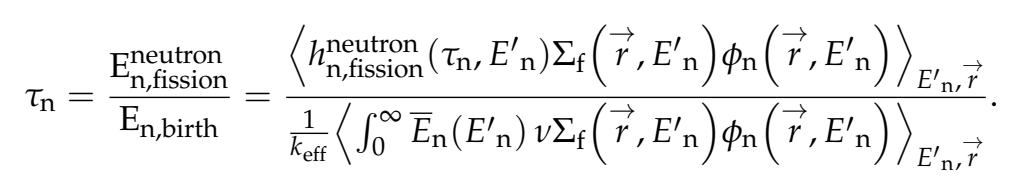

This correction factor, denoted $\tau_{\mathrm{n}}$, is referred to as the energy rebalance factor [1]. Note that Equation (10) is defined implicitly in terms of $\tau_{\mathrm{n}}$, due to the fact that the neutron energy released in fission is a function of the incident energy of the neutron causing fission. Traditionally, fission energy release data in the ENDF format includes the incident energy of the neutron causing fission, such that:

$$
h_{\mathrm{n}, \text { fission }}^{\text {total }}\left(E_{\mathrm{n}}^{\prime}\right)=Q_{\text {fission }}\left(E_{\mathrm{n}}^{\prime}\right)+E_{\mathrm{n}}^{\prime} .
$$

In order to preserve $Q_{\text {fission }}\left(E_{n}^{\prime}\right)$ it follows that the total fission energy release, $h_{\mathrm{n}, \text { fission }}^{\text {total }}\left(E_{\mathrm{n}}^{\prime}\right)$, must be adjusted in order to account for the change in the deposition energy 
of incident neutrons causing fissions in a non-critical reactor. Since the energy imbalance is the result of an artificial change in the number of neutrons released in fission, it is reasonable to assume that this adjustment is only applied to the component of fission energy released as neutrons, such that:

$$
h_{\mathrm{n}, \text { fission }}^{\text {neutron }}\left(\tau_{\mathrm{n}}, E_{\mathrm{n}}^{\prime}\right)=h_{\mathrm{n}, \text { fission }}^{\text {neutron }}\left(E_{\mathrm{n}}^{\prime}\right)+E_{\mathrm{n}}^{\prime}\left(\tau_{\mathrm{n}}-1\right) .
$$

An explicit solution for $\tau_{\mathrm{n}}$ is obtained by substituting Equation (12) into Equation (10) and rearranging to yield

$$
\tau_{\mathrm{n}}=\frac{\left\langle h_{\mathrm{n}, \text { fission }}^{\text {neutron }}\left(E_{\mathrm{n}}^{\prime}\right) \Sigma_{\mathrm{f}}\left(\vec{r}, E_{\mathrm{n}}^{\prime}\right) \phi_{\mathrm{n}}\left(\vec{r}, E_{\mathrm{n}}^{\prime}\right)\right\rangle_{E_{\mathrm{n}}^{\prime}, \vec{r}}-\left\langle E_{\mathrm{n}}^{\prime} \Sigma_{\mathrm{f}}\left(\vec{r}, E_{\mathrm{n}}^{\prime}\right) \phi_{\mathrm{n}}\left(\vec{r}, E_{\mathrm{n}}^{\prime}\right)\right\rangle_{E_{\mathrm{n}, \vec{r}}}}{\frac{1}{k_{\mathrm{eff}}}\left\langle\int_{0}^{\infty} \bar{E}_{\mathrm{n}}\left(E_{\mathrm{n}}^{\prime}\right) v \Sigma_{\mathrm{f}}\left(\vec{r}, E_{\mathrm{n}}^{\prime}\right) \phi_{\mathrm{n}}\left(\vec{r}, E_{\mathrm{n}}^{\prime}\right)\right\rangle_{E_{\mathrm{n},}^{\prime}, \vec{r}}-\left\langle E_{\mathrm{n}}^{\prime} \Sigma_{\mathrm{f}}\left(\vec{r}, E_{\mathrm{n}}^{\prime}\right) \phi_{\mathrm{n}}\left(\vec{r}, E_{\mathrm{n}}^{\prime}\right)\right\rangle_{E_{\mathrm{n}}^{\prime}, \vec{r}}} .
$$

For a thermal-spectrum-reactor, the second term in both the numerator and denominator becomes negligible. Furthermore, if the fission energy release data (ENDF MT 458) and fission spectrum data (ENDF MT 18 and 455) are consistent then $\tau_{\mathrm{n}}$ is well-approximated by $k_{\text {eff. }}$ This result is consistent with previous studies in this area, which have recommended scaling neutron energy deposition by $k_{\text {eff }}[1]$. However, Equation (13) demonstrates that the $\tau_{\mathrm{n}} \approx k_{\text {eff }}$ approximation is not accurate for reactors where a significant fraction of fissions are caused by above-thermal neutrons.

The energy rebalance factor forces consistency between neutron energy release and deposition, as defined in Equation (5). Imposing this consistency on the master energy balance equation (Equation (2)) allows cancellation of the neutron energy production and birth terms, yielding:

$$
\mathrm{Q}_{\text {indirect }}+\mathrm{Q}_{\mathrm{fission}}=\left(\mathrm{E}_{\mathrm{n} \text {,fission }}^{\text {local }}+\mathrm{E}_{\mathrm{n} \text {,fission }}^{\text {neutrino }}+\mathrm{E}_{\mathrm{n}, \text { fission }}^{\gamma}\right)+\left(\mathrm{E}_{\mathrm{n}, \text { indirect }}^{\text {local }}+\mathrm{E}_{\mathrm{n} \text {,indirect }}^{\gamma}\right)+\mathrm{E}_{\mathrm{n}, \text { leakage }} \text {. }
$$

Equation (14) is a reasonable (although not necessarily unique) interpretation of energy balance for a quasistatic system as it enforces conservation of energy release and deposition. This interpretation is based on the assumption that the rate of neutron production and loss are balanced for both mass and energy, so the net energy deposition rate only accounts for the rate at which energy is released during nuclear interactions. This implies that the amount of neutron energy present in the system remains constant between generations and does not contribute to the net energy deposition rate. The energy balance relationship shown in Equation (14) enforces conservation of $Q_{\text {fission }}$ and $Q_{\text {indirect }}$ on a per reaction basis, regardless of $k_{\text {eff. }}$. However, this does not imply that total fission or indirect energy release in the full system is independent of $k_{\text {eff }}$. In fact, the integrated (total) energy release rate in the code will necessarily change with $k_{\text {eff }}$ as the ratio of capture/scattering $\left(Q_{\text {indirect }}\right)$ to fission events $\left(Q_{\text {fission }}\right)$ varies.

\section{Self-Consistent Local Heating Edits}

The previous section established the need to distinguish between the transport energy and deposition energy for neutrons, in order to ensure balance between energy release and energy deposition (and leakage) in the reactor. However, the ultimate objective is to define local heating edits that can be used to determine the distribution of energy deposition within the reactor, while preserving energy balance.

It is a straightforward matter to use the neutron deposition energy when calculating leakage and also for adjusting the neutron energy release in fission, as described in the previous section. However, non-fission energy deposition is more complicated than leakage and fission energy deposition due to the large number of different reactions that can occur. The energy deposited for each reaction is based on energy balance where deposition is equal to the incident neutron energy plus energy released by the reaction (reaction $Q$ - 
value) minus the total energy of radiation exiting the reaction. Therefore, the total energy released/deposited during non-fission neutron/nucleus interactions is given by:

$$
E_{\mathrm{n}, \text { indirect }}^{\text {total }}=\left\langle\phi_{\mathrm{n}}\left(\vec{r}, E_{\mathrm{n}}^{\prime}\right) \sum_{i}^{\mathrm{nucs}} \sum_{j}^{\text {rxns }}\left(\tau_{\mathrm{n}} E_{\mathrm{n}}^{\prime}+Q_{i, j}\left(E_{\mathrm{n}}^{\prime}\right)-\tau_{\mathrm{n}} \bar{E}_{\mathrm{n}, i, j}\left(E_{\mathrm{n}}^{\prime}\right)\right) \Sigma_{i, j}\left(\vec{r}, E_{\mathrm{n}}^{\prime}\right)\right\rangle_{E_{\mathrm{n},}^{\prime}, \vec{r}},
$$

where $\bar{E}_{\mathrm{n}, i, j}$ is the average total energy of neutrons in the exit channel for the reaction, defined by:

$$
\bar{E}_{\mathrm{n}, i, j}\left(E_{\mathrm{n}}^{\prime}\right)=\left\langle E_{\mathrm{n}} y_{i, j}^{\mathrm{n}}\left(E_{\mathrm{n}} \mid E_{\mathrm{n}}^{\prime}\right)\right\rangle_{E_{\mathrm{n}}, E_{\mathrm{n}}^{\prime}}
$$

where $y_{i, j}^{\mathrm{n}}\left(E_{\mathrm{n}} \mid E_{\mathrm{n}}^{\prime}\right)$ is the yield density of neutrons with energy $E_{\mathrm{n}}$ due to non-fission reaction $j$ with nuclide $i$ caused by an incident neutron with pre-collision energy $E_{n}^{\prime}$. Note that we have elected to use the deposition energy for both the incident and exiting neutrons involved in the reaction. This is a somewhat arbitrary decision, since there are no constraints on how the difference between the transport and deposition energies for the neutron should be apportioned between local deposition and exiting neutrons. However, using the deposition energy for all neutrons involved in the reaction preserves the ratio of local deposition to transport energy for the neutrons, which simplifies implementation in a transport solver. Any other assumption would allow the renormalization factor to change between neutron interactions, which is cumbersome to implement for transport methods other than Monte Carlo.

The ENDF format allows for neutron-interaction heating data to be represented by a pseudoreaction cross section, referred to as KERMA (kinetic energy released in material), which is defined as [4]:

$$
K_{\mathrm{n}, \text { indirect }}^{\text {total }}\left(E_{\mathrm{n}}^{\prime}\right)=\sum_{i}^{\text {nucs rxns }} \sum_{j}^{\prime}\left(E_{\mathrm{n}}^{\prime}+Q_{i, j}\left(E_{\mathrm{n}}^{\prime}\right)-\bar{E}_{\mathrm{n}, i, j}\left(E_{\mathrm{n}}^{\prime}\right)\right) \Sigma_{i, j}\left(\vec{r}_{,} E_{\mathrm{n}}^{\prime}\right),
$$

KERMA data are computed by the NJOY HEATR module by summing over all possible reactions, including fission. However, in this work, we have assumed that fission reactions have been omitted from the KERMA definition in Equation (17). This can be accomplished by subtracting ENDF MT 458 total energy release from fission, $h_{\mathrm{n} \text {,fission }}^{\text {total }}\left(E_{\mathrm{n}}^{\prime}\right)$, data from the HEATR calculated total KERMA. Note that this correction must also account for any other potential inconsistencies between the datasets, such as the application of unresolved resonance region (URR) self-shielding factors. In general, it appears that the fission energy release (ENDF MT 458) data should be used instead of KERMA fission heating data for detailed energy deposition calculations.

Comparison of Equations (15) and (17) highlights an inconsistency between the consistent energy deposition equation and the KERMA definition. Applying the energy rebalance factor to the KERMA (Equation (17)), multiplying by flux, and integrating over volume and energy gives an approximation for the indirect energy release/deposition,

$$
\mathrm{E}_{\mathrm{n}, \text { indirect }}^{\text {total }} \approx\left\langle\phi_{\mathrm{n}}\left(\vec{r}, E_{\mathrm{n}}^{\prime}\right) \tau_{\mathrm{n}} K_{\mathrm{n}, \text { indirect }}^{\text {total }}\left(\vec{r}, E_{\mathrm{n}}^{\prime}\right)\right\rangle_{E_{\mathrm{n},}^{\prime}, \vec{r}^{\prime}}
$$

which incorrectly applies the correction factor to the $Q$-value for all reactions. This violates one of the original objectives for the self-consistent normalization technique, which was to preserve the nuclear energy release on a per reaction basis. As a result, use of the approximation in Equation (18) will result in an additive bias in the total indirect energy release. Given that indirect energy release typically accounts for approximately $1 \%$ of the total energy release in a nuclear reactor, this approximation is acceptably small in many cases. Note that this bias in the indirect energy release does not affect the balance between energy release and deposition, which will always be preserved if the energy rebalance factor is calculated from Equation (10). The correction of this bias, and the preceding ambiguity involving apportionment of deposition energy between deposition 
and secondary neutron emission, will require changes to the processing of heating data in HEATR and possibly the KERMA data format. One possible solution is to adopt a KERMA data representation that explicitly accounts for the incident neutron energy, similar to what is done for the fission energy release data (MT 458). Unfortunately, definition of an improved KERMA pseudoreaction cross section for quasistatic fission systems is beyond the scope of this paper.

\section{Numerical Results}

A simplified transport problem with an analytical reference solution for energy deposition was used to confirm the accuracy of the proposed energy-normalization technique. The simplified model involves transport of monoenergetic $2.0 \mathrm{MeV}$ neutrons in an infinite, homogenous mixture of two purely-absorbing nuclides: fuel and poison. The fuel nuclide is purely-fissioning, with $\sigma_{\mathrm{t}}=\sigma_{\mathrm{f}}=1.0$. Each fission event releases 2.0 neutrons and $200 \mathrm{MeV}$ of energy (190 MeV excluding neutrinos), divided among charged particles, neutrons, photons, and neutrinos as shown in Table 1 . The poison nuclide is purely-absorbing, with $\sigma_{\mathrm{t}}=\sigma_{\mathrm{a}}=1.0$ and a reaction $Q$-value of $1.0 \mathrm{MeV} /$ absorption. In all tests, the number density of the fuel nuclide was held constant at $N_{\mathrm{f}}=1.0$ atoms $/(\mathrm{barn} \cdot \mathrm{cm})$, while the number density of the poison nuclide, $N_{\mathrm{p}}$, was adjusted from 0.5 to 1.5 atoms / (barn $\left.\cdot \mathrm{cm}\right)$ in order to vary the multiplication factor of the system.

Table 1. Fission energy release by category for fictitious, purely-fissioning fuel nuclide.

\begin{tabular}{|c|c|c|}
\hline Category & $\begin{array}{c}\text { Nominal Energy Rel. }(h(0)) \\
{[\mathrm{MeV} / \text { Fiss }]}\end{array}$ & $\begin{array}{c}\text { Energy-Dependent Energy Rel. } \\
\left(h\left(E^{\prime}\right)\right) \\
{[\mathrm{MeV} / \text { Fiss }]}\end{array}$ \\
\hline Fission Fragments $\left(h^{\mathrm{FR}}\right)$ & 169.0 & $h^{\mathrm{FR}}(0)$ \\
\hline Prompt Neutrons ${ }^{\dagger}\left(h^{\text {neutron }}\right)$ & 1.386 & $h^{\text {neutron }}(0)+1.307 \times E^{\prime} \times\left(\tau_{\mathrm{n}}-1\right)$ \\
\hline Prompt Gammas ${ }^{\dagger}\left(h^{\gamma}\right)$ & 12.15 & $h^{\gamma}(0)-0.075 \times E^{\prime}$ \\
\hline Beta Particles $\left(h^{\beta}\right)$ & 7.15 & $h^{\beta}(0)-0.075 \times E^{\prime}$ \\
\hline Neutrinos $\left(h^{\text {neutrino }}\right)$ & 10.2 & $h^{\text {neutrino }}(0)-0.010 \times E^{\prime}$ \\
\hline
\end{tabular}

${ }^{\dagger}$ Delayed neutron and photon release values are assumed to be equal to zero.

Due to the simplistic nature of this test problem, it is possible to analytically solve for the energy release and deposition as a function of poison density, and thus, the multiplication factor for the system. The multiplication factor is given by:

$$
k_{\mathrm{eff}}=\frac{N_{\mathrm{F}} v \sigma_{\mathrm{f}}}{N_{\mathrm{F}} \sigma_{\mathrm{f}}+N_{\mathrm{P}} \sigma_{\mathrm{a}}}=\frac{2}{1+N_{\mathrm{P}}}
$$

In keeping with the fundamental assumption that energy release per reaction should be preserved independently of the multiplication factor, it follows:

$$
\frac{\mathrm{E}_{\mathrm{n} \text {,deposited }}^{\text {total }}}{\text { fission }}=\frac{\mathrm{Q}_{\text {total }}}{\text { fission }}=\frac{\mathrm{Q}_{\text {fission }}^{\text {sensible }}}{\text { fission }}+\frac{N_{\mathrm{P}} \sigma_{\mathrm{a}}}{N_{\mathrm{F}} \sigma_{\mathrm{f}}} \frac{\mathrm{Q}_{\text {indirect }}}{\text { absorption }}=190+N_{\mathrm{P}} \frac{\mathrm{MeV}}{\text { fission }}
$$

As noted previously, the objective of self-consistent energy normalization is to preserve energy release per reaction $\left(Q_{\text {fission }}\right.$ and $\left.Q_{\text {indirect }}\right)$, while also preserving balance between energy release and energy deposition, regardless of the multiplication factor for the system. Analytical energy release and deposition values for the test problem were calculated for three different energy normalization schemes: no energy renormalization $(\tau=1)$, approximate renormalization ( $\tau=k_{\text {eff }}$ ), and exact renormalization, using $\tau$ as defined in Equation (10). In addition, the effect of applying the renormalization factor to $Q_{\text {indirect }}$ (Equation (18)) was considered for the approximate and exact renormalization techniques. The accuracy of each normalization approach was assessed by determining the ratio of the total energy deposition to the reference solution (Equation (20)) as well as the ratio of 
total energy deposition to total energy release, over a range of poison nuclide densities (multiplication factors). Analytical results for all cases are shown in Figures 2 and 3.

These results in Figures 2 and 3 demonstrate that the exact renormalization scheme (Equation (10)) preserves balance between energy release and deposition for all multiplication factors. In addition, the exact renormalization agrees with the reference energy release/deposition solution as long as the energy rebalance factor is not applied to the $\mathrm{Q}_{\text {indirect }}$ component of local neutron energy deposition. Scaling local energy deposition (including $Q_{\text {indirect }}$ ) by the energy rebalance factor produces a positive bias in total energy release/deposition by a factor of $N_{\mathrm{P}} \cdot \mathrm{Q}_{\text {indirect }}$, which reaches a relative energy deposition error of $\pm 0.2 \%$ at $k_{\text {eff }}=1.333$ and 0.8 , respectively.

The results without energy renormalization or with the approximate $\left(\tau=k_{\text {eff }}\right)$ renormalization scheme exhibit a clear bias trend in energy deposition, as well as a corresponding imbalance between energy release and deposition rates for non-critical systems, as expected. If no correction is applied, then the neutron energy deposition bias is equal to the deviation from criticality $\left(1-k_{\text {eff }}\right)$ multiplied by the ratio of fission energy released as neutrons to the total fission energy release rate $\left(h_{\text {neutron }} / h_{\text {total }}\right)$. In this example problem, the unnormalized energy deposition exhibits a bias of $\pm 0.2 \%$ over the range $k_{\text {eff }}=0.8$ to 1.333 . The use of the approximate energy renormalization factor reduced the energy deposition bias by approximately a factor of 2 , but does not eliminate it completely. This is because the approximate renormalization factor does not account for the effect of the quasistatic approximation on the energy of the incident neutrons causing fission, which is appreciable in this fast-spectrum example.

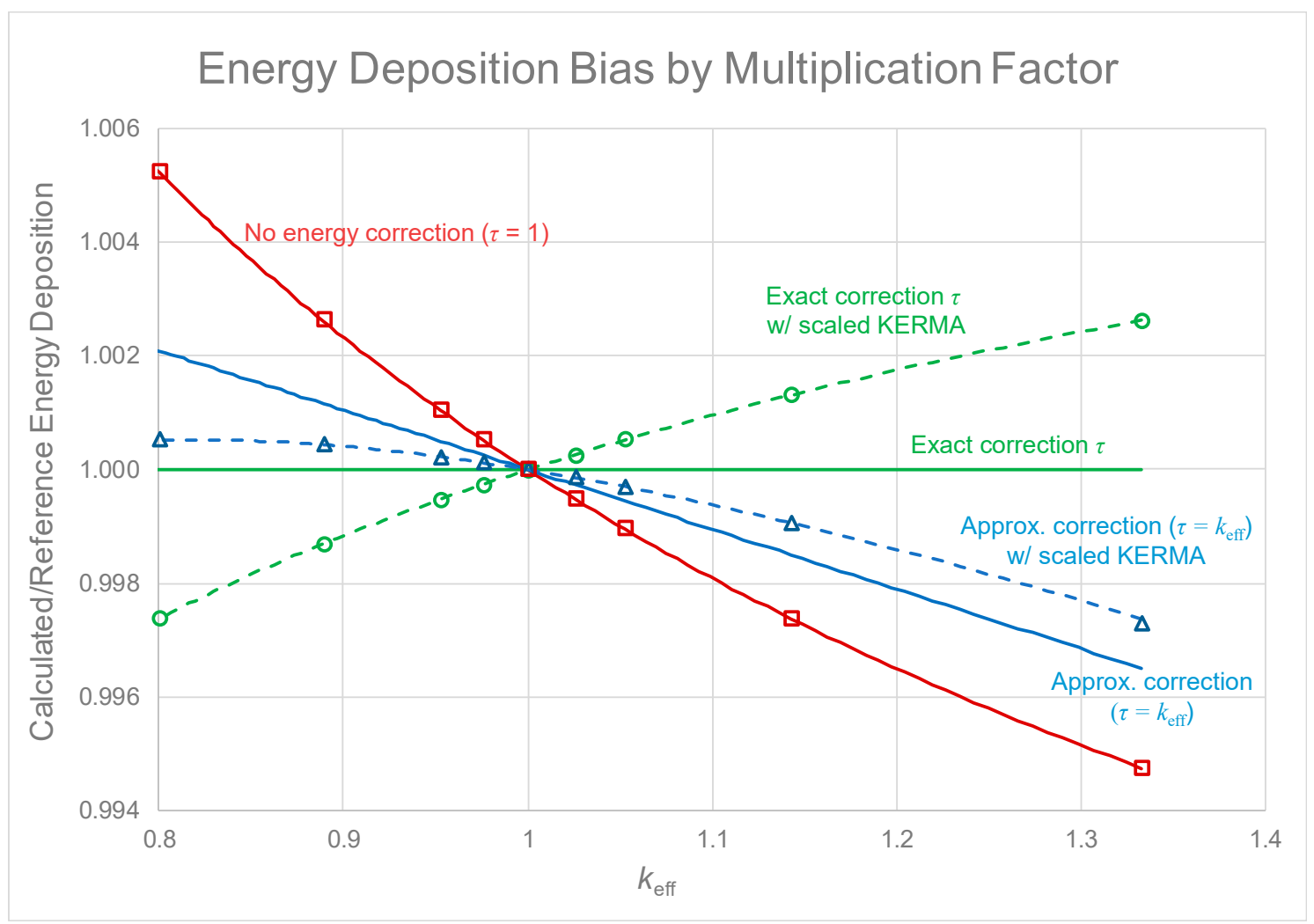

Figure 2. Energy deposition bias as a function of multiplication factor for an infinite, homogeneous, two-nuclide test problem. Theoretical results are provided for three energy correction schemes (no correction, approximate, and exact), and for reference (solid lines) and scaled KERMA values (dashed lines). Corresponding numerical results from an MC21 calculation are shown as open markers for selected conditions. 


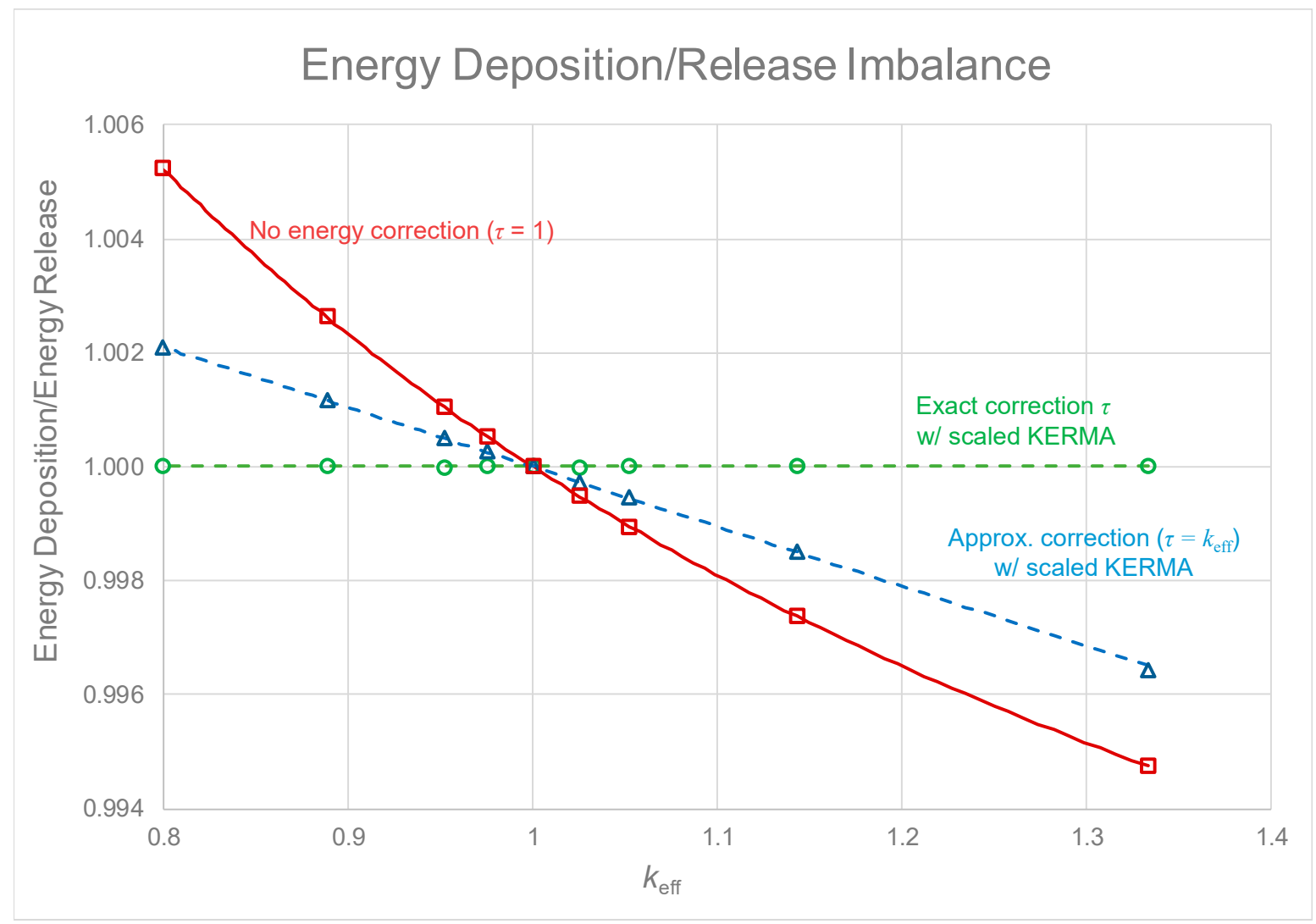

Figure 3. Energy deposition to release imbalance as a function of multiplication factor for an infinite, homogeneous, two-nuclide test problem. Theoretical results are provided for three energy correction schemes (no correction, approximate, and exact), and for reference (solid lines) and scaled KERMA values (dashed lines). Corresponding numerical results from an MC21 calculation are shown as open markers for selected conditions.

In order to demonstrate the energy imbalance effect in a real radiation transport solver, the MC21 Monte Carlo code [5] was used to calculate energy deposition in the example problem. The simulation used 110 batches (10 discard) of 10,000 histories, with a manually-created nuclear data library containing data for the two fictitious nuclides defined previously in this Section. MC21 results for the energy deposition bias and energy deposition/release imbalance for nine different criticality conditions show excellent agreement with the theoretical predictions, as illustrated in Figures 2 and 3 (open data markers). Note that all MC21 results use the KERMA scaling approximation due to the previously-discussed limitations in the representation of KERMA data in the ENDF format and the corresponding use of the data by transport solvers.

\section{Conclusions}

The quasistatic $\left(k_{\text {eff }}\right)$ approximation commonly used for fission criticality calculations will necessarily lead to an imbalance between energy release and energy deposition for non-critical systems. In this paper, we formally quantified this imbalance and presented a normalization factor for energy deposition that preserves nuclear energy release per reaction, as well as enforcing energy balance between release and deposition mechanisms, regardless of the criticality state of the system. The normalization technique distinguishes between the transport energy of a neutron, used for evaluating reaction cross sections, and the corresponding deposition energy, which is available for the transfer to the surrounding material. Implementation of the correction technique involves the application of an energy rebalance factor to local energy deposition or transfer quantities, including leakage, fission neutron emission, and local energy deposition (KERMA). Theoretical and numerical results are presented, which demonstrate that the self-consistent method is able to preserve 
reaction energy release and energy release/deposition balance. However, limitations in the ENDF format for local energy deposition (KERMA) data prevent conservation of indirect energy release for most applications. This issue warrants further investigation and may be mitigated by the establishment of an improved representation of KERMA data in the ENDF format.

Author Contributions: The first author named is lead and corresponding author. Contributions to this work are listed below, using the CRediT taxonomy. Conceptualization, D.P.G., S.J.D. and M.H.S.; methodology, D.P.G. and S.J.D.; software, D.P.G.; validation, D.P.G., S.J.D. and M.H.S.; formal analysis, D.P.G. and S.J.D.; investigation, D.P.G., S.J.D. and M.H.S.; writing-original draft preparation, D.P.G.; writing-review and editing, D.P.G., S.J.D. and M.H.S. All authors have read and agreed to the published version of the manuscript.

Funding: This research received no external funding.

Data Availability Statement: The authors confirm that the data supporting the findings of this study are available within the article.

Acknowledgments: This manuscript has been authored by Fluor Marine Propulsion under contract No. DOE-89233018CNR000004 with the U.S. Department of Energy. The United States government retains and the publisher, by accepting this article for publication, acknowledges that the United States Government retains a non-exclusive, paid-up, irrevocable, and world-wide license to publish, distribute, translate, duplicate, exhibit, and perform the published form of this manuscript, or allow others to do so, for United States Government purposes.

Conflicts of Interest: The authors declare no conflict of interest. The funders had no role in the design of the study; in the collection, analyses, or interpretation of data; in the writing of the manuscript, or in the decision to publish the results.

\section{References}

1. Griesheimer, D.P.; Stedry, M.H. A Generalized Framework for In-Line Energy Deposition during Steady-State Monte Carlo Radiation Transport. In Proceedings of the International Conference on Mathematics and Computational Methods Applied to Nuclear Science and Engineering (M\&C 2013), Sun Valley, ID, USA, 5-9 May 2013.

2. Tuominen, R.; Valtavirta, V.; Leppänen, J. New Energy Deposition Treatment in the Serpent 2 Monte Carlo Transport Code. Ann. Nucl. Energy 2019, 129, 224-232.

3. Trkov, A.; Herman, M.; Brown, D.A. ENDF-6 Formats Manual. In BNL-90365-2009 Rev. 2; Brookhaven National Laboratory: Upton, NY, USA, 2011.

4. Kahler, A.C. The NJOY Nuclear Data Processing System; Version 2012. LA-UR-12-27079; Los Alamos National Laboratory: Los Alamos, NM, USA, 2013.

5. Griesheimer, D.P.; Gill, D.F.; Nease, B.R.; Sutton, T.M.; Stedry, M.H.; Dobreff, P.S.; Carpenter, D.C.; Trumbull, T.H.; Caro, E.; Joo, H.; et al. MC21 v.6.0-A Continuous-Energy Monte Carlo Particle Transport Code with Integrated Reactor Feedback Capabilities. Ann. Nucl. Energy 2015, 82, 29-40. [CrossRef] 